\title{
Perfil epidemiológico dos idosos brasileiros que buscam tratamento fisioterapêutico: uma revisão de literatura
}

\author{
Epidemiological profile of elderly brazilians seeking physical therapy treatment: a literature review \\ Perfil epidemiológico de ancianos brasileños que buscan tratamiento fisioterapéutico: revisión de la
}

\section{literatura}

Recebido: 27/09/2021 | Revisado: 30/09/2021 | Aceito: 01/10/2021 | Publicado: 03/10/2021

\author{
Adiele Sousa da Costa \\ ORCID: https://orcid.org/0000-0002-5769-5566 \\ Centro Universitário da Amazônia, Brasil \\ E-mail: adiele.jc@hotmail.com \\ Ana Carolina Branches dos Santos \\ ORCID: https://orcid.org/0000-0002-8343-0131 \\ Centro Universitário da Amazônia, Brasil \\ E-mail:anabranches12@gmail.com \\ Gabriela Carolina Marcião Seixas de Souza \\ ORCID: https://orcid.org/0000-0002-5894-0607 \\ Centro Universitário da Amazônia, Brasil \\ E-mail:gabrielacarolina884@yahoo.com \\ Indiara de Alencar \\ ORCID: https://orcid.org/0000-0003-3204-3278 \\ Universidade do Estado do Pará, Brasil \\ E-mail: indiara.alencarstm@gmail.com
}

\begin{abstract}
Resumo
O processo de envelhecimento ocorre durante a vida afetando todos os organismos, sendo considerada de forma progressiva causando diversas alterações dos padrões fisiológicos do indivíduo, envolvendo fatores sociais, culturais, biológicos e psicológicos. A fisioterapia pode ser necessária em qualquer fase da vida, porém para a população idosa ela é indispensável não só relacionado ao tratamento, mas preventiva, contribuindo para melhor qualidade de vida. Desta forma, é extremamente importante identificar o perfil dos pacientes atendidos numa dada população, conhecer os usuários do serviço de fisioterapia, entender a demanda do serviço, e patologias mais frequentes. Trata-se de uma revisão de literatura exploratória descritiva, método pelo qual se narra detalhadamente um tema estudado em pesquisas anteriores, com o intuito de abranger mais sobre determinado assunto. A pesquisa foi realizada nos seguintes bancos de dados da área da saúde: Google Acadêmico, Literatura Latino-Americana e do Caribe em Ciências da Saúde (LILACS) e Scientific Eletronic Library Online (SciELO). Nesta pesquisa foi possível observar uma crescente busca por atendimento fisioterapêutico em diversas áreas de atuação, sendo que, a predominância dos pacientes atendidos era do sexo feminino com idade superior a 60 anos. Outro dado importante observado na pesquisa foi o fato que os pacientes não possuíam apenas um diagnóstico, mais várias comorbidades diminuindo a capacidade funcional do idoso causando efeitos deletérios na sua qualidade de vida, vale ressaltar que a necessidade de atendimentos mais frequentes pelos fisioterapeutas fora provocada por distúrbios musculares representando $83 \%$ dos casos e distúrbios neurológicos com 61,1\% de buscas por atendimentos.
\end{abstract}

Palavras-chave: Fisioterapia; Idoso; Envelhecimento; Epidemiologia.

\begin{abstract}
The aging process occurs during life, affecting all organisms, being considered progressively causing several changes in the individual's physiological patterns, involving social, cultural, biological and psychological factors. Physical therapy may be necessary at any stage of life, but for the elderly population it is essential not only related to treatment, but preventive, contributing to a better quality of life. Thus, it is extremely important to identify the profile of patients seen in a given population, know the users of the physiotherapy service, understand the service demand, and the most frequent pathologies. This is a descriptive exploratory literature review, a method by which a topic studied in previous research is narrated in detail, in order to cover more about a given subject. The search was carried out in the following healthcare databases: Academic Google, Latin American and Caribbean Health Sciences Literature (LILACS) and Scientific Electronic Library Online (SciELO). In this research, it was possible to observe a growing search for physiotherapeutic care in several areas of activity, and the predominance of patients seen was female over 60 years of age. Another important fact observed in the research was the fact that patients did not have only one diagnosis, but several comorbidities, decreasing the functional capacity of the elderly, causing deleterious effects on their quality of life, it is noteworthy that the need for
\end{abstract}


more frequent care by physical therapists was caused by muscle disorders representing $83 \%$ of cases and neurological disorders with $61.1 \%$ of searches for assistance.

Keywords: Physiotherapy; Old man; Aging; Epidemiology.

\begin{abstract}
Resumen
El proceso de envejecimiento ocurre durante la vida, afectando a todos los organismos, considerándose progresivamente provocando varios cambios en los patrones fisiológicos del individuo, involucrando factores sociales, culturales, biológicos y psicológicos. La fisioterapia puede ser necesaria en cualquier etapa de la vida, pero para la población anciana es fundamental no solo relacionada con el tratamiento, sino preventiva, contribuyendo a una mejor calidad de vida. Por ello, es de suma importancia identificar el perfil de los pacientes atendidos en una población determinada, conocer a los usuarios del servicio de fisioterapia, conocer la demanda del servicio y las patologías más frecuentes. Se trata de una revisión bibliográfica exploratoria descriptiva, método mediante el cual se narra en detalle un tema estudiado en investigaciones anteriores, con el fin de abarcar más sobre un tema determinado. La búsqueda se realizó en las siguientes bases de datos de salud: Google Académico, Literatura de Ciencias de la Salud de América Latina y el Caribe (LILACS) y Biblioteca Electrónica Científica en Línea (SciELO). En esta investigación se pudo observar una creciente búsqueda de cuidados fisioterapéuticos en varias áreas de actividad, y el predominio de pacientes atendidos fue del sexo femenino mayor de 60 años. Otro hecho importante observado en la investigación fue el hecho de que los pacientes no tenían un solo diagnóstico, sino varias comorbilidades, reduciendo la capacidad funcional de los ancianos, provocando efectos deletéreos en su calidad de vida, se destaca la necesidad de cuidados más frecuentes por fisioterapeutas fue causado por trastornos musculares que representan el $83 \%$ de los casos y trastornos neurológicos con el $61,1 \%$ de las búsquedas de asistencia.
\end{abstract}

Palabras clave: Fisioterapia; Anciano; Envejecimiento; Epidemiología.

\title{
1. Introdução
}

O envelhecimento é um processo natural do ser humano que acomete países tanto desenvolvido, quanto aqueles que estão em desenvolvimento como o Brasil (Santos, et al., 2013). Este ocorre de forma individual e em ritmos diversos e diferenciados em comparado a idade cronológica de cada um. Segundo a Organização Mundial de Saúde (OMS), os países desenvolvidos consideram idoso cuja idade seja igual ou maior que 65 anos, diferente dos países em desenvolvimento que consideram idosos pessoas com idade superior ou igual a 60 anos. (Bardon, et al., 2016).

O processo de envelhecimento ocorre durante a vida afetando todos os organismos, sendo considerada de forma progressiva resultando em diversas alterações dos padrões fisiológicos de um indivíduo, envolvendo fatores sociais, culturais, biológicos e psicológicos. O conceito de envelhecer é compreendido como inúmeras alterações biopsicossociais que transformar aspectos comuns em indivíduos sadios, levando-os a novas ideias de enfrentamento da vida (Cunha, et al., 2019).

O aumento da população idosa deve ocorrer com qualidade de vida, visto que, com o passar da idade, elevam os casos relacionados à problemática das Doenças Crônicas não transmissíveis demonstrando um grau elevado de morbimortalidade no Brasil. (Duncan, et al., 2012). As doenças crônicas não transmissíveis (DCNT) de maior impacto na saúde pública constituem as doenças cardiovasculares, câncer, diabetes mellitus, e as doenças respiratórias crônicas. Estas representam aproximadamente $80 \%$ das DCNT. Vale ressaltar que é significativo os casos de doenças reumáticas, causando maior impacto na saúde pública brasileira (Malta, et al., 2011).

O Estatuto do Idoso regulamentado pela Lei 10.741 de $1^{\circ}$ de outubro de 2003, assegura a atenção integral à saúde, por intermédio do Sistema Único de Saúde (SUS), assim como lhe é garantido a acesso universal e igualitário em conjunto articulado e contínuo das ações e serviços para a prevenção, promoção, proteção e recuperação da saúde (Freitas, 2010).

De acordo com Conselho Federal de Fisioterapia e Terapia Ocupacional (COFFITO), a fisioterapia possibilita ações aplicadas à promoção e recuperação da saúde de seus pacientes. Ela é a ciência da área de saúde que estuda, previne e trata às transformações cinético-funcionais que acometem os órgãos e sistemas do corpo humano com origem em inúmeras causas. (COFFITO, 2019).

O fisioterapeuta é considerado um profissional generalista, sendo capaz de atuar em todos os níveis de atenção básica, primária, secundária e terciário. A fisioterapia pode ser necessária em qualquer fase da vida, porém para a população idosa ela se torna indispensável não só relacionado ao tratamento, mas preventiva, contribuindo para melhor qualidade de vida. Uma das 
principais metas da fisioterapia é ajudar o indivíduo a adquirir maior independência funcional, tendo em consideração sua potencialidade e limitações, e por consequência retardar ou evitar danos posteriormente (Passos \& Lima, 2019).

Devido às mudanças atuais na estrutura etária da população, a epidemiologia do envelhecimento decorre de uma melhor compreensão do processo de envelhecimento. No campo da saúde do idoso, a epidemiologia considera sua cultura, gênero, ambiente natural e determinantes de saúde para analisar o estado de saúde da população e se esforça para determinar, descrever e medir suas capacidades funcionais, qualidade de vida, status socioeconômico, e grau de independência, além de determinar que as doenças associadas é causa de morte e morbidade da população, também organiza a atenção à saúde do idoso (Basso, 2013).

Desta forma, é de grande relevância identificar o perfil dos pacientes atendidos numa dada população, conhecer os usuários do serviço de fisioterapia, entender a demanda do serviço assim também como as patologias mais frequentes. Com base nessa concepção, é possível criar e tracejar uma assistência específica e própria que recepcione as exigências e respeite suas peculiaridades, para assegurar um cuidado especializado (Costa, et al, 2020). O presente estudo tem como objetivo traçar o perfil epidemiológico do idoso que busca tratamento fisioterapêutico.

\section{Metodologia}

A presente pesquisa trata-se de uma revisão de literatura exploratória descritiva, e estratégia qualitativa, onde se narra detalhadamente um tema estudado em pesquisas anteriores, com o intuito de abranger mais sobre determinada temática. (Rother, 2007).

A pesquisa foi desenvolvida obedecendo aos seguintes passos: identificação do tema; criação de critérios de inclusão e exclusão; criação e seleção dos estudos; análise e apreciação dos resultados. Para conduzir a elaboração dessa revisão determinamos como a questão problema: "Qual o perfil epidemiológico do idoso que busca tratamento fisioterapêutico?"

Foram selecionados como embasamento para a pesquisa, materiais já publicados sobre o tema em livros, artigos científicos e revistas, disponíveis nos seguintes bancos de dados da área da saúde: Google Acadêmico, Literatura Latino-Americana e do Caribe em Ciências da Saúde (LILACS) e Scientific Eletronic Library Online (SciELO), utilizando os descritores: fisioterapia; idoso e envelhecimento.

Como critérios de inclusão foram selecionados artigos que abordassem sobre o tema escolhido, publicados entre o período de 2015 a 2021 e na língua portuguesa. Os critérios de exclusão foram artigos que não possuem relação com o tema, publicações anteriores ao ano de 2015 e trabalhos incompletos.

Após a análise minuciosa dos artigos que contemplavam os filtros das pesquisas, foram utilizados 10 artigos que condiziam com o tema pesquisado nesta revisão de literatura.

\section{Resultados e Discussão}

No Quadro 1 mostra-se 10 estudos realizados com ambos os gêneros, totalizando 8508 idosos que procuraram atendimento fisioterapêutico e que foram submetidos aos estudos para verificar o perfil dessa população. 
Quadro 1 - Resultados dos estudos entre os anos 2015 a 2021.

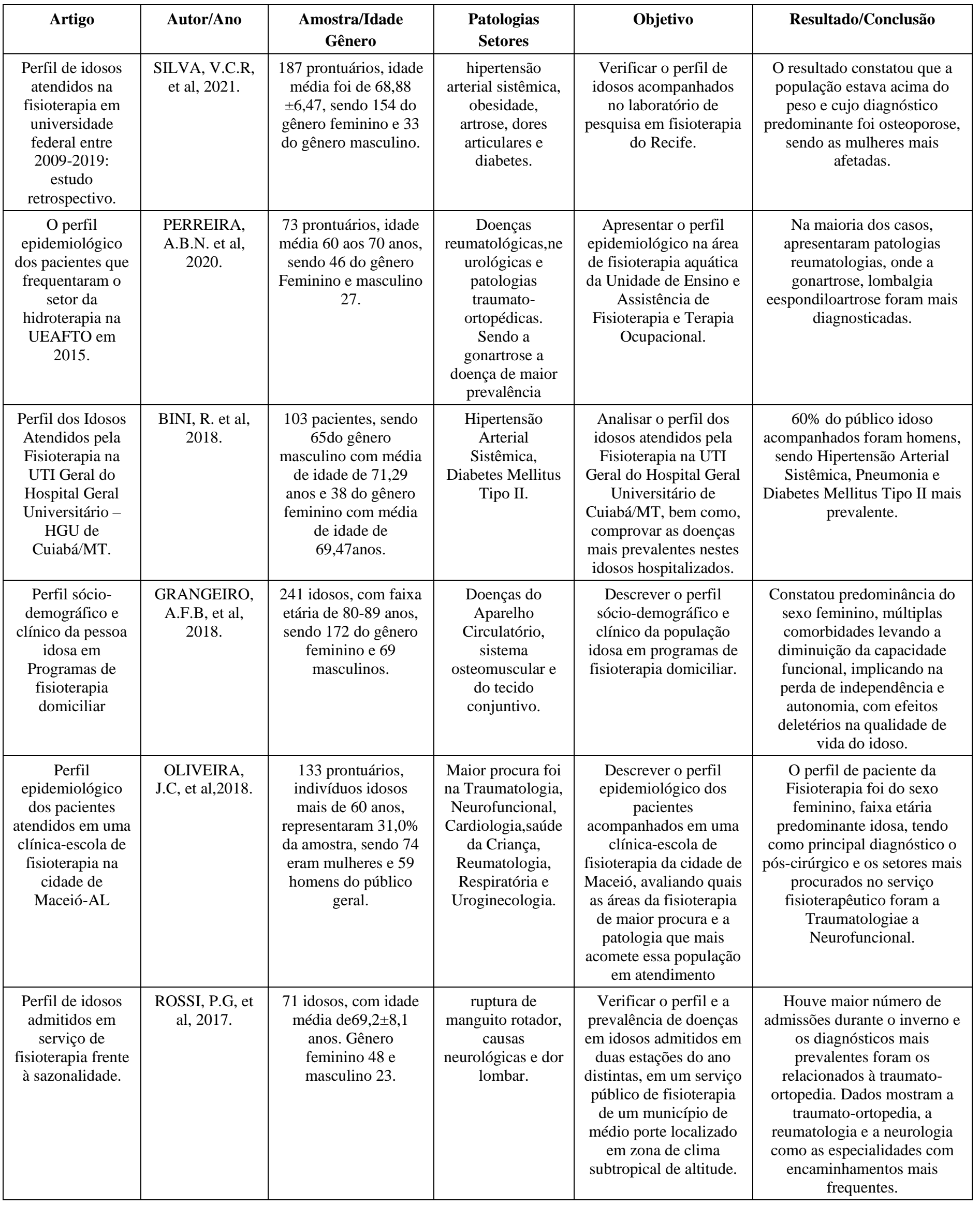




\begin{tabular}{|c|c|c|c|c|c|}
\hline $\begin{array}{c}\text { Caracterização } \\
\text { clínica e } \\
\text { epidemiológica } \\
\text { dos pacientes em } \\
\text { atendimento } \\
\text { domiciliar na } \\
\text { cidade de } \\
\text { Maceió, AL, } \\
\text { Brasil. }\end{array}$ & $\begin{array}{l}\text { CARNAÚBA, } \\
\text { C.M.D, et al., } \\
2017 .\end{array}$ & $\begin{array}{l}859 \text { pacientes, sendo } \\
445 \text { do serviço } \\
\text { público e } 414 \text { do } \\
\text { serviço privado. Com } \\
\text { idade média } 72,62 \\
( \pm 18,28), \text { sendo } 489 \\
\text { do gênero feminino e } \\
370 \text { masculinos }\end{array}$ & $\begin{array}{c}\text { doenças } \\
\text { neurológicas, } \\
\text { oncológicas, } \\
\text { endócrinase } \\
\text { ortopédicas. } \\
\text { Sendo o Acidente } \\
\text { vascular } \\
\text { encefálico mais } \\
\text { acometido }\end{array}$ & $\begin{array}{c}\text { Retratar clínica e } \\
\text { epidemiologicamente } \\
\text { pessoas em atendimento } \\
\text { domiciliar no município } \\
\text { de Maceió, AL, Brasil. }\end{array}$ & $\begin{array}{c}\text { Alguns resultados são } \\
\text { divergentes ao estabelecer } \\
\text { uma comparação entre serviço } \\
\text { público e serviço privado, a } \\
\text { maioria dos pacientes } \\
\text { recebendo tratamento a } \\
\text { assistência domiciliar é para } \\
\text { idosos, acamados, do gênero } \\
\text { feminino, com doenças } \\
\text { neurológicas, sendo o } \\
\text { diagnóstico mais comum de } \\
\text { AVE. }\end{array}$ \\
\hline $\begin{array}{l}\text { Estudo } \\
\text { epidemiológico } \\
\text { sobre acidente } \\
\text { vascular } \\
\text { encefálico em } \\
\text { uma clínica } \\
\text { escolar de } \\
\text { fisioterapia. }\end{array}$ & $\begin{array}{c}\text { MARTINS, } \\
\text { E.R.C, et } \\
\text { al,2016. }\end{array}$ & $\begin{array}{l}212 \text { questionários, a } \\
\text { média geral de idade } \\
\text { foi de } 62,7 \pm 13,83 \\
\text { anos, sendo do gênero } \\
\text { feminino } 116 \text { e } \\
\text { masculino } 96 .\end{array}$ & $\begin{array}{l}\text { AVE isquêmico e } \\
\text { hemorrágico. }\end{array}$ & $\begin{array}{c}\text { Conhecer o perfil } \\
\text { epidemiológico dos } \\
\text { pacientes com sequelas } \\
\text { de Acidente vascular } \\
\text { encefálico que } \\
\text { buscaram atendimento } \\
\text { na Clínica Escola de } \\
\text { Fisioterapia da } \\
\text { UNICENTRO, no } \\
\text { município de } \\
\text { Guarapuava/PR. }\end{array}$ & $\begin{array}{l}\text { O AVE prevalente foi o } \\
\text { isquêmico, no sexo masculino, } \\
\text { com lado esquerdo mais } \\
\text { afetado. O maior número de } \\
\text { casos foi na faixa etária entre } \\
\text { 70-79 anos. A hiperlipidemia } \\
\text { e HAS foram os principais } \\
\text { fatores de risco. }\end{array}$ \\
\hline $\begin{array}{l}\text { Prevalência de } \\
\text { encaminhamentos } \\
\text { às doenças } \\
\text { musculoesqueléti } \\
\text { cas segundo a } \\
\text { classificação } \\
\text { estatística } \\
\text { internacional de } \\
\text { doenças (CID- } \\
\text { 10): reflexões } \\
\text { para formação do } \\
\text { fisioterapeuta na } \\
\text { área de } \\
\text { musculoesqueléti } \\
\text { ca. }\end{array}$ & $\begin{array}{c}\text { SOUZA, C.S, et } \\
\text { al,2015. }\end{array}$ & $\begin{array}{c}6.516 \\
\text { encaminhamentos, a } \\
\text { idade média de } 40 \text { aos } \\
70 \text { anos, sendo o sexo } \\
\text { feminino }(70,52 \%) \\
\text { com } \\
\text { encaminhamentos e } \\
\text { masculino }(29,48 \%) \text {. }\end{array}$ & $\begin{array}{l}\text { Dor lombar baixa, } \\
\text { síndrome do } \\
\text { manguito rotador } \\
\text { e a gonartrose } \\
\text { não-identificada. }\end{array}$ & $\begin{array}{l}\text { Detalhar o perfil das } \\
\text { pessoas encaminhada } \\
\text { aos serviços de } \\
\text { Fisioterapia e } \\
\text { procedimentos } \\
\text { pertencentes a } \\
\text { Classificação Estatística } \\
\text { Internacional de } \\
\text { Doenças, CID-10, na } \\
\text { cidade de Ribeirão } \\
\text { Preto e fornecer } \\
\text { reflexões para a } \\
\text { formação profissional } \\
\text { no Brasil. }\end{array}$ & $\begin{array}{l}\text { O estudo demonstra que os } \\
\text { diagnósticos de condições } \\
\text { musculoesqueléticas para } \\
\text { atendimento fisioterapêutico } \\
\text { mais referido foram a dor } \\
\text { lombar baixa, a síndrome do } \\
\text { manguito rotador e a } \\
\text { gonartrose não-especificada } \\
\text { em ambos os sexos, sendo } \\
\text { predominantemente mulheres } \\
\text { e a maior parte dos } \\
\text { procedimentos } \\
\text { fisioterapêuticos foram } \\
\text { solicitados a serviços de nível } \\
\text { secundário e terciário. }\end{array}$ \\
\hline
\end{tabular}

Fonte: Costa, Santos, Souza e Alencar (2021).

Na presente pesquisa foi possível observar uma crescente busca por atendimento fisioterapêutico em diversas áreas de atuação, sendo que, a predominância dos pacientes atendidos era do sexo feminino com idade superior a 60 anos. Outro dado importante observado na pesquisa foi o fato que os pacientes não possuíam apenas um diagnóstico, mais sim várias comorbidades diminuindo a capacidade funcional do idoso causando efeitos deletérios na sua qualidade de vida, vale ressaltar que a necessidade de atendimentos mais frequentes pelos fisioterapeutas fora provocada por distúrbios musculares apresentando $83 \%$ dos casos e distúrbios neurológicos com $61,1 \%$ de buscas por atendimentos. 
No estudo realizado por Silva et al 2021, observou-se que a osteoporose teve maior prevalência quanto ao diagnóstico, seguido de algias articulares e disfunções posturais, foi constatado que $40 \%$ da amostra encontrava-se acima do peso e estavam associados a outra comorbidades como hipertensão arterial sistêmica e artrose. Já no estudo de Pereira et al 2020, a patologia mais prevalente foram as doenças reumáticas $(83 \%)$ seguidas das neurológicas (10\%), vale ressaltar que nas duas pesquisas houve predominância no sexo feminino com idade entre 60 e 70 anos. O estudo de Bini et al 2018, apontou a hipertensão Arterial Sistêmica como a doença de maior prevalente com (32\%) dos casos com faixa etária de 71,29 anos, seguida de Diabetes de Mellitus Tipo II. Vale enfatizar que houve maior incidência em pessoas do gênero masculino (60\%), com a justificativa que o gênero masculino buscar menos os serviços de atenção primária em relação ao sexo feminino. Diferente da pesquisa de Grangeiro et al 2018, constatou que as doenças com maior predominância são aquelas que acometem o aparelho circulatório, osteomuscular e tecido conjuntivo, sendo mais frequentes no sexo feminino com idade média de 80 a 89 anos, com agravantes de múltiplas comorbidades implicando na perda de independência e autonomia dos acometidos.

No estudo de Oliveira et al 2018, mostrou maior prevalência de atendimentos em pessoas do sexo feminino $(55,6 \%) \mathrm{em}$ relação ao sexo masculino com idades entre mais que 60 anos, representando 31,0\% da amostra total. Quanto a patologia mais frequente, o estudo mostrou o pós-cirúrgico na área de cardiologia representando $(82,6 \%)$ dos casos, quanto as áreas de fisioterapia com maior índice de tratamento, a pesquisa evidenciou maior procura na traumatologia (33,8\%), Neuro-funcional (20,3\%), Cardiologia (11,3\%). Na pesquisa de Carnaúba et al 2017, observou-se maior predominância em doenças neurológicas $(61,1 \%)$, sendo o Acidente Vascular Encefálico a causa mais frequente de atendimento (35,2\%), com idade média de 72,62 com maior predominância do sexo feminino.

Segundo o autor Rossi et al 2017, mostrou que o diagnóstico com maior prevalência nos atendimentos estão relacionados à traumato-ortopédico e reumatologia com maior predominância no gênero feminino e idade média de 69,2 anos, isso se justifica devido clima frio, pois a hipotermia atua no enrijecimento articular e muscular causando doenças reumáticas e ortopédicas, já o estudo de Martins et al 2016que aplicou 212 questionários, observou o acidente vascular encefálico isquêmico (14,1\%) e hemorrágico $(7,6 \%)$ com maior prevalência nos atendimentos, vale ressaltar que a hiperlipidemia e pressão arterial sistêmica são os principais fatores de risco para essas patologias, nesse estudo foi verificado maior prevalência no gênero masculino com idade superior a 60 anos.

Conforme a pesquisa de Rezende et al 2015, a predominância dos atendimentos eram em idosos do gênero feminino com idade superior a 59 anos. As patologias mais frequentes durante o atendimento foram a gonartrose com $28,9 \%$ dos casos, seguida de fibromialgia (13,7\%) e artrose na coluna vertebral (12,7\%). Já o estudo de Sousa et al 2015, mostrou maiores atendimentos em doenças músculo esqueléticas como a dor lombar baixa (17,99\%) seguida pela síndrome do manguito rotador $(6,57 \%)$ e gonartrose não identificada $(6,11 \%)$. Notou-se que a frequência dos atendimentos s concentra na faixa etária dos 40 aos 79 anos de idade, sendo mais frequentes em mulheres.

Para obtenção dos resultados da pesquisa, seis autores obtiveram os dados a partir dos prontuários de atendimentos, porém, vale ressaltar que esse método demonstra escassez de informações que dificulta a análise da pesquisa, pois muitos desses dados são repassados de forma incompleta causando incoerência nos resultados. Contudo, a pesquisa de Grangeiro et al 2018 utilizou como instrumentos na coleta de dados, a caderneta de saúde da pessoa idosa, a qual é disponibilizada pelo Ministério da Saúde, já o estudo de Sousa et al 2015 obteve os resultados através de encaminhamentos fisioterapêuticos.

\section{Conclusão}

No presente estudo foi possível analisar que durante o processo do envelhecimento, a fisioterapia é de suma importância tanto na prevenção como no tratamento de patologias específicas do envelhecimento. O objetivo da intervenção fisioterapêutica é 
sempre preservar ou restaurar a funcionalidade, autonomia e independência da pessoa idosa, levando sempre em consideração aspectos de origem biológica, psicológica e social de cada indivíduo.

Pode se analisar que a busca por atendimento fisioterapêutico é crescente no sexo feminino com idades entre 60 a 89 anos, onde o auto cuidado é maior no público feminino devido às mesmas serem mais propensas ao acometimento de patologias crônicas, diferente do público masculino que procura menos pelo atendimento primário de saúde.

Em relação ao estado de saúde dos idosos, a maioria apresentava mais de um diagnóstico sendo os mais frequentes: distúrbios musculares, pós-operatório cardíaco e doenças neurológicas. Durante a pesquisa foi observado que os pacientes atendidos apresentavam também outras comorbidades principais como hipertensão arterial sistêmica, diabetes de mellitus tipo II e obesidade.

Conclui-se que o estudo do perfil epidemiológico do idoso é necessário para orientar efetivamente o fisioterapeuta a realizar pesquisas prévias sobre determinadas patologias e problemáticas com o público-alvo de cada área realizando assim o tratamento adequado conforme a necessidade de cada indivíduo.

Devido à escassez de artigos já publicados sobre a temática, recomenda-se novos estudos que abordem de forma mais ampla sobre o perfil epidemiológico dos idosos que procuram atendimento fisioterapêutico, com isso sendo possível conhecer quanto á idade, sexo, quais as patologias que mais acometem os idosos, possibilitando um melhor planejamento para um tratamento adequado, assim melhorando a qualidade de vida deles.

\section{Agradecimentos}

Agradecemos imensamente à Deus, por ter nos concedido saúde, força e disposição para superarmos todos os obstáculos.

\section{Referências}

Basso, A. (2013). O perfil epidemiológico de idosos atendidos pela estratégia de saúde da família do município de Bossoroca-RS. Trabalho de Conclusão de Curso (Especialização em Informação Científica e Tecnológica em Saúde) - Instituto de Comunicação e Informação Científica e Tecnológica em Saúde, Fundação Oswaldo Cruz, Porto Alegre.

Bardon, F. J. et al. (2016). Alterações celulares no envelhecimento humano. Journal of Oral Investigations - JOI, 5(1), 61-65.

Bini, R. et al. (2018). Perfil dos Idosos Atendidos pela Fisioterapia na UTI Geral do Hospital Geral Universitário - HGU de Cuiabá/MT. Jornal of Health Sciences, 20(1), 25-28.

Carnaúba, C. M. D. et al. (2017). Caracterização clínica e epidemiológica dos pacientes em atendimento domiciliar na cidade de Maceió, AL, Brasil. Revista Brasileira de Geriatria e Gerontologia, 20(3), 353-363.

COFFITO. (2021) - Leis e decretos. Conselho Federal de Fisioterapia e Terapia Ocupacional (COFFITO). http://coffito.gov.br/nsite/?p=3318.

Costa, L. E. B. R \& Silva, M. A. (2020). Perfil epidemiológico de pacientes atendidos pela fisioterapia: uma revisão de literatura. Trabalho de Conclusão de Curso (Graduação em Fisioterapia) - Centro Universitário CESMAC de Maceió - AL.

Cunha, J. S. et al. (2019). Produção Científica Acerca da Assistência de Enfermagem no Envelhecimento Saudável da Pessoa Idosa- Faculdade de Enfermagem São Vicente de Paula, João Pessoa, edição especial, 159 a 176.

Duncan, B.B. et al. (2012). Doenças crônicas não transmissíveis no Brasil: prioridade para enfrentamento e investigação. Revista Saúde Pública, 46(1), 126-134.

Freitas, L. D. O. (2010). O processo de envelhecimento natural da pele do idoso: diagnostico e intervenção de enfermagem. Trabalho de Conclusão de Curso (Graduação Enfermagem) - Universidade Federal do Rio Grande do Sul.

Grangeiro, A.F.B. et al. (2018). Perfil sociodemográfico e clínico da pessoa idosa em Programas de Fisioterapia Domiciliar. O Mundo Da Saúde, 42(3), 656-677.

Martins, E.R.C. et al. (2016). Estudo epidemiológico sobre acidente vascular encefálico em uma clínica escola de Fisioterapia. Espaço Para Saúde, 17(1), 32-38.

Malta, D. C. et al. (2011). Apresentação do plano de ações estratégicas para o enfrentamento das doenças crônicas não transmissíveis no Brasil, 2011 a 2022. Epidemiologia e Serviços de Saúde, 20(4), 425-438.

Oliveira, J. C. et al. (2018). Perfil epidemiológico dos pacientes atendidos em uma clínica - escola de fisioterapia na cidade de Maceió-AL. Interfaces Científicas Saúde E Ambiente, 6(2), 85-94.

Passos, L. M. S. C. \& Lima, M. P. D. (2019). Fisioterapia preventiva para melhoria da qualidade de vida dos idosos do Município de Conceição do Canindé - PI. Trabalho de Conclusão de Curso (Especialização em Curso de Especialização de Saúde da Família e Comunidade) - Universidade Federal do Piauí. 
Research, Society and Development, v. 10, n. 13, e31101321113, 2021

(CC BY 4.0) | ISSN 2525-3409 | DOI: http://dx.doi.org/10.33448/rsd-v10i13.21113

Pereira, A. B. N. et al. (2020). O perfil epidemiológico dos pacientes que frequentaram o setor da hidroterapia na UEAFTO em 2015. Brazilian Journal of Health Review, 3(6), 18813-18817.

Rezende, A. L. C. et al. (2015). Perfil epidemiológico dos pacientes atendidos na clínica escola de fisioterapia do Uniaraxá no setor de hidroterapia. Revista Odontológica de Araçatuba, 36(2), 09-13.

Rossi, P. G. et al. (2017). Perfil de idosos admitidos em serviços de fisioterapia frente à sazonalidade. Revista Scientia Médica, 27(2), 1-5.

Rother, E. T. (2017). Revisão sistemática X revisão narrativa. Acta Paulista de Enfermagem, 20(2), 5-6.

Santos, V. C. F. et al. (2013). Perfil das internações por doenças crônicas não-transmissíveis sensíveis à atenção primária em idosos da metade sul do RS. Revista Gaúcha Enfermagem, 34(3), 124-131.

Silva, V. C. R. et al. (2021). Perfil de idosos atendidos na fisioterapia em universidade federal entre 2009-2019 estudo retrospectivo. Saúde e Pesquisa, 14(4), 8806 .

Souza, C. S. et al. (2015). Prevalência de encaminhamentos às doenças musculoesqueléticas segundo a classificação estatística internacional de doenças (CID-10): reflexões para formação do fisioterapeuta na área de musculoesquelética. Fisioterapia e Pesquisa, 22(1), 48-53. 